\title{
ESTUDOS GEOFÍSICOS INTEGRADOS NO LIXÃO DE CUIABÁ, MT, BRASIL - RESULTADOS PRELIMINARES
}

Shozo Shiraiwa, ${ }^{1}$ Solange Pereira Lisovsky, Vagner Roberto Elis, ${ }^{2}$ Jorge Luís Porsani, ${ }^{3}$ Welitom Rodrigues Borges ${ }^{4}$

Recebido em 23 ago., 2002 / Aceito em 13 dez., 2003

Received Aug. 23, 2002 / Accepted Dec. 13, 2003

\section{ABSTRACT}

One of the major concerns in geoenvironmental projects is the soil and water contamination caused by the waste disposal. The geophysical methods applied are a good way to get some information required in a site characterization program. This work shows some results obtained through resistivity, EM inductive and GPR surveys in the search of informations about the impact caused by a waste disposal area in Cuiabá, State of Mato Grosso, Brazil.

Keywords: GPR-Ground Penetrating Radar, Electrical Resistivity, Electromagnetic Induction, Landfill, Cuiabá, MT, Brazil.

\section{RESUMO}

Uma das maiores preocupaçães em projetos geoambientais é o estudo da contaminaç̧̃o dos solos e da água subterrânea causada pela disposição de lixo urbano. Os métodos geofísicos aplicados são um bom caminho para pegar algumas informaç̃ōes requeridas num programa de caracterização. Este trabalho mostra alguns resultados obtidos através de levantamentos de resistividade, eletromagnético indutivo e GPR (Ground Penetrating Radar), na busca de informaç̃oes sobre 0 impacto causado pela área de disposição do lixo em Cuiabá, Estado do Mato Grosso, Brasil.

Palavras-chave: Resistividade elétrica, Eletromagnético indutivo, GPR-Ground Penetrating Radar, Contaminação do meio ambiente, lixão de Cuiabá.

Departamento de Geologia-NEGEA-UFMT - Av. Fernando Corrêa da Costa, s/n - Bairro Boa Esperança - Cuiabá - MT - CEP: 78060-000 - Tel.: (65) 615-8745 - Fax: (0xx65) 615-8737 E-mail: shozo@cpd.ufmt.br

2 IAG-USP - Departamento de Geofísica - Rua do Matão 1226 - Cidade Universitária - São Paulo - SP - CEP: 05508-090 - Tel.: (11) 30914734 - Fax: $0 x x 1130915034$ E-mail: vagnelis@iag.usp.br

3 IAG-USP - Departamento de Geofíisica - Rua do Matão 1226 - Cidade Universitária - São Paulo - SP - CEP: 05508-090 - Tel.: (11) 30914734 - Fax: $0 x x 1130915034$ - E-mail: porsani@iag.usp.br

4 Pós-Graduação em Geofísica-IAG/USP - Rua do Matão, 1226 - Cidade Universitária - São Paulo-SP - CEP: 05508-090 - Tel.: (11) 3091-2792 - Fax: (0xx11) 3091 -5034 E-mail: welitom@iag.usp.br 


\section{INTRODUÇÃOO}

0 processo migratório em busca de melhorias é um dos fatores do aumento da produção de lixo urbano, comprometendo todo 0 planejamento urbano. Em apenas três décadas 0 Brasil reverteu a relação entre população urbana e população rural. Enquanto até o final da década de 1960 havia $30 \%$ das pessoas morando nas cidades e as demais na zona rural, hoje temos $70 \%$ de nossos habitantes vivendo em zonas urbanas. 0 conhecimento técnico do espaço físico, normalmente, não é levado em conta para a escolha de locais adequados para a disposição final dos resíduos sólidos, e com isso tem-se a formação de "lixões". Um dos problemas gerados com a disposição inadequada dos resíduos urbanos é a contaminação do lençol freático. Contudo, os problemas poderiam ser amenizados com a utilização de métodos geofísicos para escolha de áreas apropriadas e na monitoração do depósito. Os métodos de Eletroresistividade, Em Indutivo e GPR mostram-se eficientes na localização e no monitoramento da contaminaç̧o das águas subterrâneas, sobretudo considerando-se a rapidez e o baixo custo com que são obtidas as informações. Buscando mostrar os principais problemas do destino final dos resíduos sólidos em depósitos a céu aberto, denominados "lixões", foi escolhido como objeto deste estudo o antigo "Lixão" de Cuiabá. Esse depósito, hoje desativado, encontra-se às margens do córrego Camundongo, que deságua no Ribeirão do Lipa, afluente do rio Cuiabá, a 12 km do centro da cidade (ELIS; MATTOS; CUTRIM, 1995).

\section{CARACTERÍSTICAS GEOLÓGICAS E HIDROGEOLÓGICAS}

A área de estudo está inserida no Grupo Cuiabá, que é formado por rochas metamórficas de baixo grau, de idade Pré-Cambriana. Migliorini, Ruiz e Santos (1998) definem três associações litológicas nas regiões de Cuiabá e Várzea Grande: (1) metadiamictitos, (2) metaortocomglomerados e metarenitos grossos quartzosos, com filito subordinados e (3) filitos sericíticos laminados ou maciços com raras intercalações de metarenitos feldspáticos. Na associação (1) os veios de quartzo são raros e subconcordantes com a foliação principal $\left(S_{1}\right)$, com atitude entre N40-50E/ 40-60NW, as juntas dominantes são subparalelas às foliações $S_{2}$ (N40-50E / 50-70SE) e $S_{3}$ (N40-50W / 75-85NE ou SW), esta última mais comum. A associação (2) distinguese pelo grande volume de veios de quartzo, principalmente nos conglomerados e arenitos. Foram distinguidas pelo menos cinco gerações de veios de quartzo: veios paralelos à $S_{0}$ e $S_{1}$ dobrados: veios tabulares paralelos à $S_{1} / S_{0}$ : veios en echelon, veios discordantes conjugados $e$ veios tabulares discordantes. As diáclases são mais abundantes nos metapelitos e agrupam-se em duas famílias principais, paralelas a $S_{2}$ e $S_{3}$. Na associaçãa (3) os veios são poucos, centimétricos e definem três famílias: veios dobrados em $D_{1}$, veios tabulares e subparalelos a $S_{1}(\mathrm{NE})$ e $S_{3}$ como também pelos tipos rochosos "conglomerados e arenitos grossos" e os "diamictitos com matriz arenosa grossa", que formam os melhores aquíferos". Os sistemas de diáclases e veios de quartzo que recortam as rochas do grupo Cuiabá, exercem papel relevante no fluxo e armazenamento das águas subterrâneas na região de estudo. Segundo Migliorini (1999), o sistema aqǘfero na região de Cuiabá e Várzea Grande é do tipo livre, heterogêneo e anísotrópico, com intensa variação lateral e em profundidade.

\section{INVESTIGAÇÕES REALIZADAS E RESULTADOS}

Dentro de uma proposta de aplicação integrada de métodos geofísicos para caracterização do problema ambiental originado pelo Lixão de Cuiabá, foram realizadas investigaç̧óes geofísicas, tais como, Sondagem Elétrica Vertical (SEV's), Caminhamento Eletromagnético (EM) e Perfis de Radar de Penetração no Solo - GPR (Figura 1). As SEV's foram adquiridas com arranjo Schlumberger e objetivaram estudar 0 perfil vertical do terreno, individualizando as camadas de solo, a zona saturada, o topo do filito inalterado, bem como a camada de resíduos e a presença de contaminação nos pontos investigados dentro do depósito de lixo propriamente dito. Os perfis de caminhamento EM foram adquiridos com 0 equipamento EM34 de forma a investigar as profundidades teóricas de 7,5, 15 e 30 metros. Esses perfis foram realizados visando delimitar a área ocupada por resíduos e identificar a zona de influência da contaminação. Os perfis GPR objetivaram imagear a subsuperfície rasa, visando localizar a pluma de contaminação, a profundidade do nível freático, a estratigrafiia do lixão e localizar regiões anômalas dentro dos resíduos.

A análise conjunta das SEV's, perfis EM e perfis de GPR permitiram a caracterização da área quanto à profundidade da zona saturada e do topo da rocha, existência e espessura da camada de resíduos e contaminaç̃õo do lençol freático pelo chorume gerado. Essa análise pôde ser realizada através dos perfis das Linhas 2 e 5 . A Linha 2 está posicionada em uma zona de borda da área ocupada pelos resíduos, e possibilitou uma visualização das condições do local com pouca influência da contaminação. A Figura 2 mostra a seçãa geológica construída por meio dos resultados obtidos das SEV's, da seção de condutividade aparente obtida pelo EM e do perfil GPR, permitindo observar 0 comportamento da zona saturada, do topo do filito e da presença de resíduos e influência de contaminaç̃ão. A zona saturada é caracterizada 


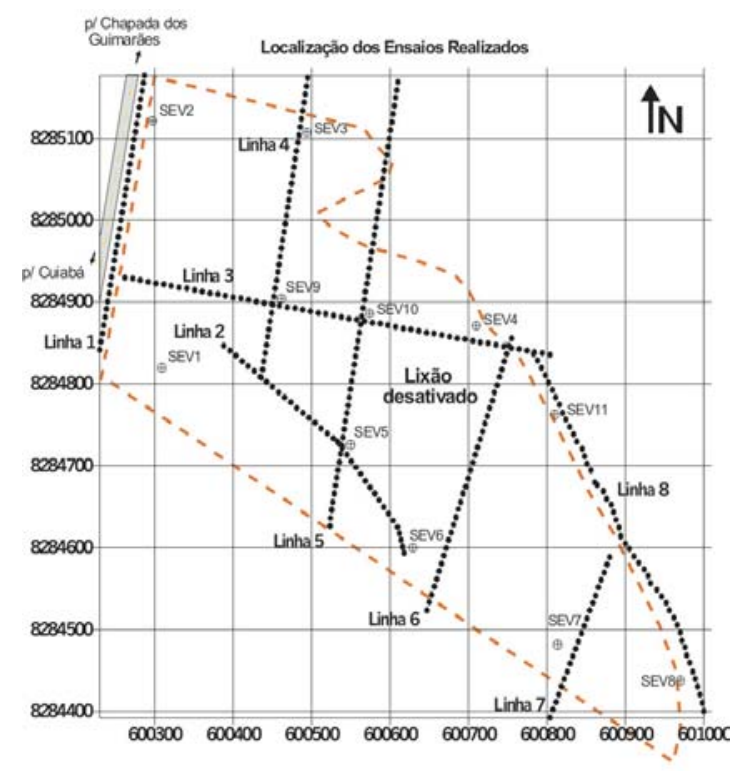

Figura 1 - Localização das investigações geofísicas na área do Lixão de Cuiabá.

Figure 1- Location of the geophysical investigations in the Cuiabá landfill.

pela camada com valores de resistividade entre 16.4 a 44 ohm.m que ocorre em profundidades de 5 a 10 metros. 0 topo do filito está abaixo dessa camada, a profundidades de 20 a 30 metros, com resistividades de 293 a 437 ohm.m. A seção de condutividade mostra em sua grande parte valores de condutividade aparente relativamente baixos, de 4 a 10 $\mathrm{mS} / \mathrm{m}$ (valores de resistividade aparente de 250 e 100 ohm.m, respectivamente) e uma zona mais condutora superficial entre 260 e 280 metros, com condutividades de até $22 \mathrm{mS} / \mathrm{m}$ (45,5 ohm.m). Essa situação aliada às informações do perfil GPR, onde ocorre uma zona com refletores localizados também próximos da posição 260 metros, sugere a presença de restos de material metálico misturado com resíduos nessa posição. Informações sobre o local sugerem a possível presença de restos de carteiras escolares. 0 refletor subhorizontal em torno de $6 \mathrm{~m}$ de profundidade, pode estar relacionado com o topo do nível freático.

A Linha 5 atravessa 0 depósito transversalmente, o que pode ser visualizado na seção de condutividade aparente (Figura 3). No início do perfil ocorre uma zona mais condutora $(60 \mathrm{mS} / \mathrm{m})$ provavelmente relacionada com a presença de resíduos. 0 depósito de lixo principal ocorre das posições 160 a 360 do perfil, com valores de condutividade aparente de 60 a $180 \mathrm{mS} / \mathrm{m}$ (resistividades de 16,6 a 5,6 ohm.m, respectivamente). Pode-se notar a presença dessa zona condutora em profundidade, sugerindo a presença de contaminação na zona saturada que está posicionada a menos de 10 metros da superfície. A SEV10, realizada sobre a camada de resíduos, mostra que essa tem espessura de cerca de 8 metros com resistividade de 5,3 ohm.m. Da posição 360 até 0 final da seção, os valores de condutividade aparente são ainda mais altos que 0 background, que estão relacionados ao encontro da zona contaminada por chorume invadindo a planície de inundação de um córrego de regime intermitente localizado na posição 460 do perfil. Inclusive a presença de uma zona mais condutora vertical nessa posição sugere fortemente que esse córrego está encaixado em uma zona de fratura. No perfil GPR pode-se notar a presença de chorume dentro dos resíduos da posição 210 até o final do perfil, pela atenuação quase total do sinal.

\section{CONCLUSÕES PRELIMINARES}

Os resultados mostram que a interpretação integrada geofísicageológica permite caracterizar a área do lixão e, conseqüentemente, a pluma de contaminação resultante do chorume originado. De acordo com os resultados, a pluma contaminante atinge a zona saturada. Da interpretação das Sondagens Elétricas Verticais, foi possível caracterizar a zona de transição solo-rocha, definir a profundidade do topo rochoso, a profundidade do nível d'água e a espessura do pacote de lixo. Esses resultados são reflexo da disposição inadequada dos resíduos, em local onde o nível freático é pouco profundo e nas proximidades de um córrego, de forma que ocorre 0 transporte de chorume para outros locais onde existem moradias de populações ribeirinhas, as quais convivem com 0 


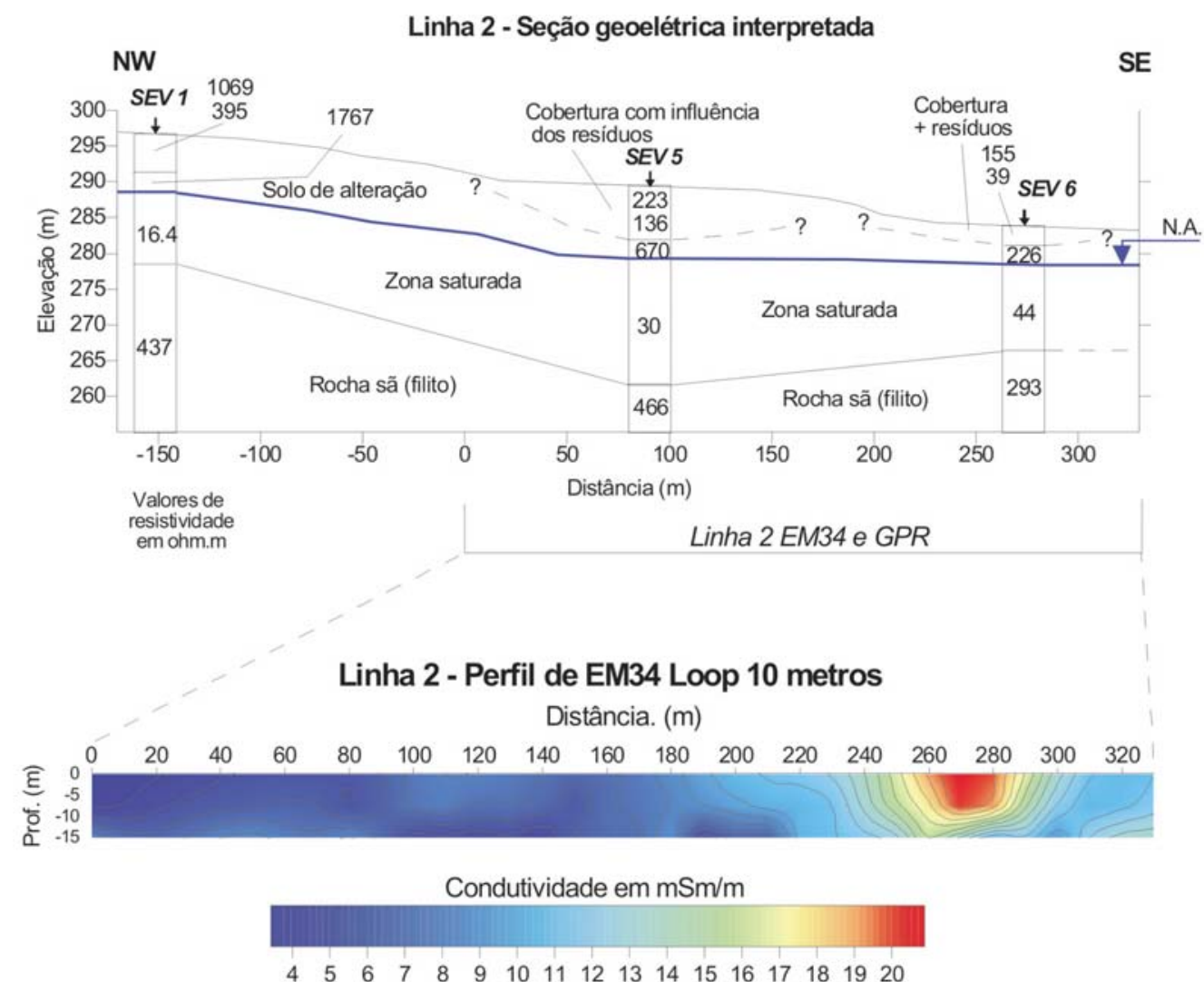

Linha 2 Perfil GPR freq. $=100 \mathrm{MHz}$

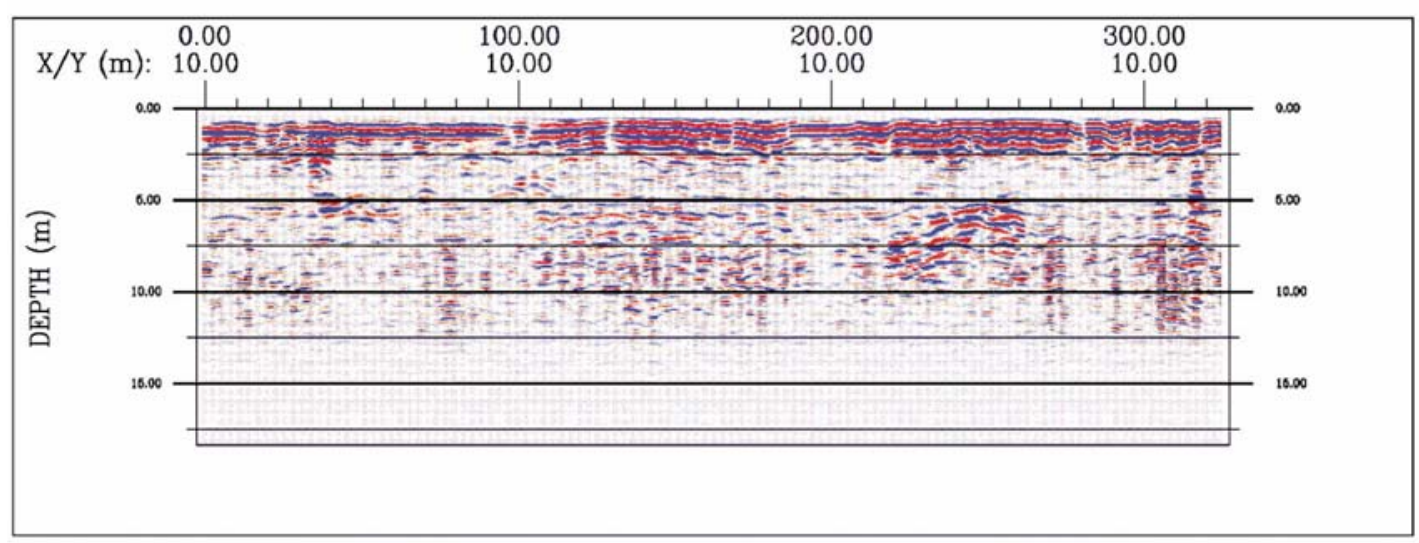

Figura 2 - Interpretação geofísica integrada da Linha 2.

Figure 2-Geophysical interpretation of the Line 2. 


\section{Linha 5 - Seção geoelétrica interpretada}

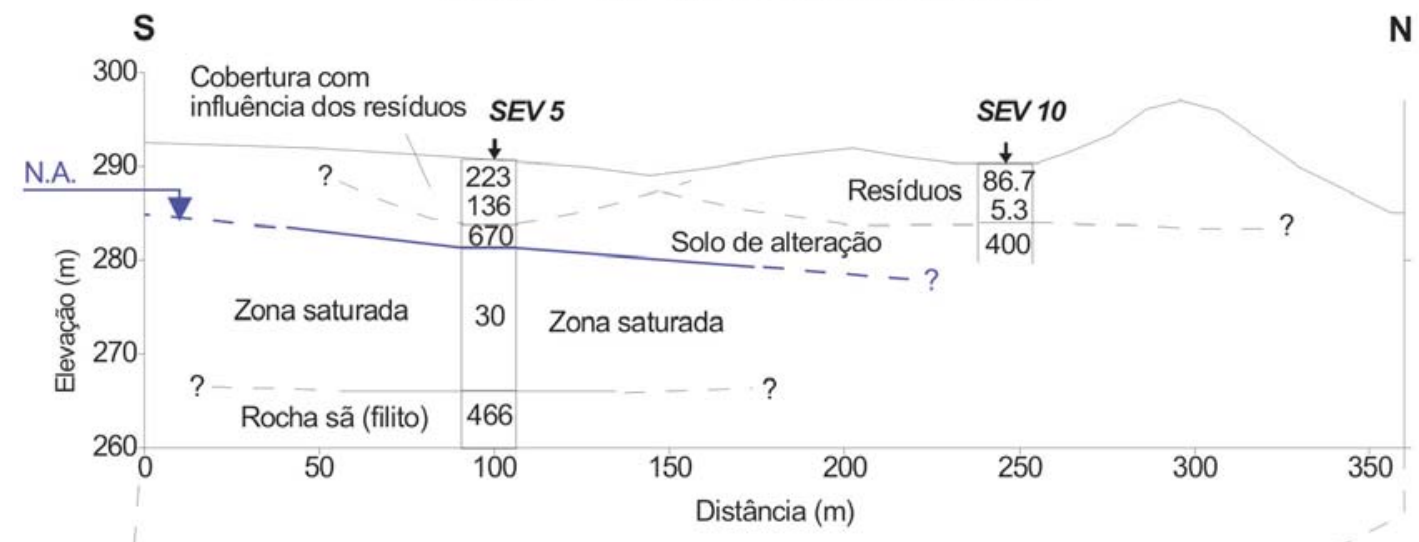

Linha 5 - Perfil EM34 Loop 10 e 20 metros

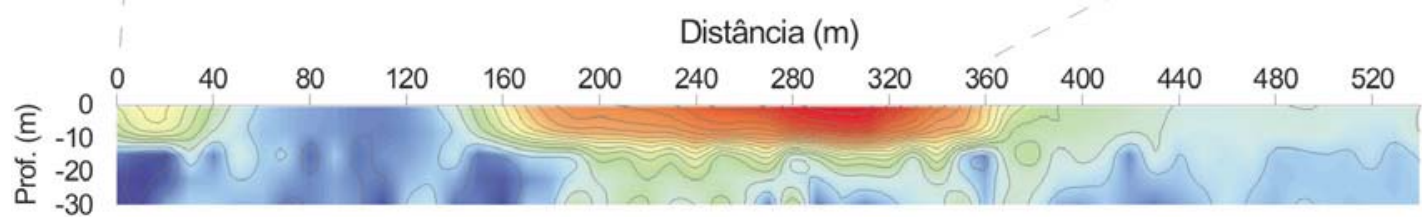

Condutividade Aparente (mS/m)

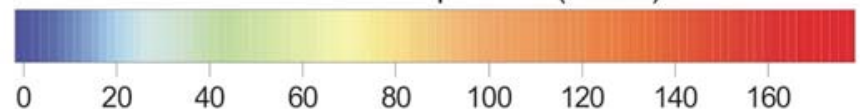

Linha 5 Perfil GPR freq. $=50 \mathrm{Mhz}$

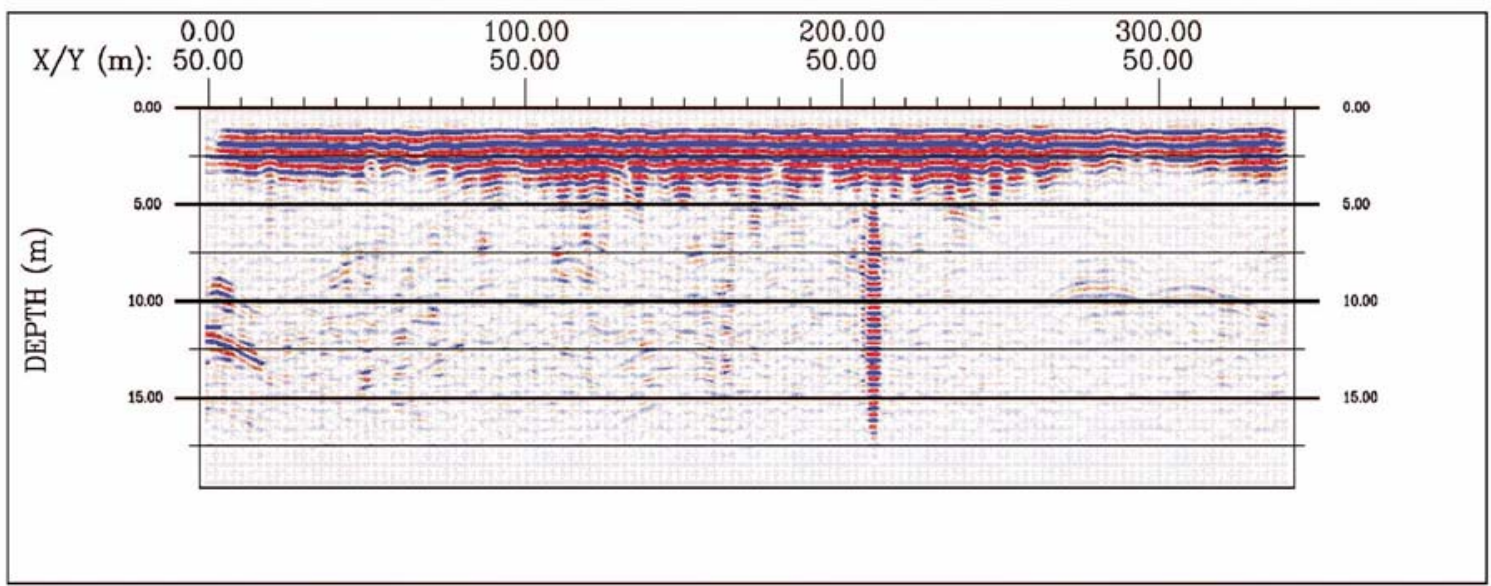

Figura 3 - Interpretação geofísica integrada da Linha 5.

Figure 3-Geophysical interpretation of the Line 5. 
perigo da contaminação. 0 problema mais grave detectado neste local, além da própria evolução da pluma, é que essa população utiliza-se de cacimbas perfuradas nos limites do avanço da pluma, que num futuro próximo poderá ter conseqüências danosas à saúde.

\section{REFERÊNCIAS}

ELIS, V. R.; MATTOS, M. R.; CUTRIM, A. O. Sondagens elétricas aplicadas ao estudo da contaminação gerada pelo lixão de Cuiabá - MT. In:
SIMPÓSIO DE GEOLOGIA DO CENTRO-0ESTE, 5., 1995, Goiânia. Anais... Goiânia: SBG/Núcleo Centro-Oeste, 1995. p. 37-39.

MIGLIORINI, R. B.; RUIZ, A. S.; SANTOS, A. A. Caracterização dos sistemas de diáclases e dos veios de quartzo no grupo Cuiabá. Regiões de Cuiabá e Várzea Grande - MT. In: ENCONTRO DE INICIAÇÃO CIENTíFICA, 6., 1998, Cuiabá, Anais...Cuiabá: PROPEP/UFMT, 1998. p. 77

. Hidrogeologia em meio urbano. Região de Cuiabá e Várzea

Grande - MT. 1999. Tese (Doutorado)-Universidade de São Paulo, São Paulo, 1999.

\section{NOTAS SOBRE OS AUTORES}

Shozo Shiraiwa licenciou-se em 1976, em Física, pela Universidade de São Paulo (USP). Desde então é professor do Departamento de Física do Instituto de Ciências Exatas e da Terra da Universidade Federal de Mato Grosso (ICET/UFMT). Mestre (1985) e Doutor (1994) em Geofísica pelo Instituto de Astronomia, Geofísica e Ciências Atmostéricas (IAG-USP), leciona e orienta alunos nos cursos de Graduação em Geologia e Pós-Graduação em Física e Meio Ambiente do ICET/UFMT. Desenvolve vários projetos com métodos gravimétricos, elétricos e eletromagnéticos aplicados à geologia, geotecnia, meio ambiente e arqueologia. Atualmente é coordenador do Núcleo de Geofísica e Estudos Ambientais da UFMT.

Vagner Roberto Elis é graduado em Geologia (1990) pela Universidade Estadual Paulista. Mestre em Geociências pela Universidade Estadual Paulista, em 1993, desenvolvendo a dissertação a "A aplicação da geofísica para análise do meio físico: Importância para elaboração de mapeamento geotécnico". Doutor em Geociências pela Universidade Estadual Paulista, em 1999, defendendo a tese "Avaliação da aplicabilidade de métodos elétricos de prospecção geofísica no estudo de áreas utilizadas para disposição de resíduos". Docente do Departamento de Geofísica do Instituto de Astronomia, Geofísica e Ciências Atmosféricas da Universidade de São Paulo, com atuação nas áreas de Geofísica Aplicada a estudos ambientais e hidrogeológicos.

Jorge Luís Porsani é geólogo pelo Instituto de Geociências da UFBa (1987). Mestrado em Geofísica pelo Núcleo de Pesquisas Geofísicas Aplicadas à Prospecção de Hidrocarbonetos da UFPa (1991). De 1991 a 1996, trabalhou como Geofísico no Centro de Pesquisas da PETROBRAS. Doutorado em Geociências e Meio Ambiente pelo Instituto de Geociências e Ciências Exatas da UNESP (1999). Desde 01/12/ 1998 é docente do Departamento de Geofísica do IAG/USP, atuando com métodos geoelétricos aplicados à geologia, geotecnia, meio ambiente e arqueologia. Atualmente, é vice coordenador do Programa de Pós-Graduação em Geofísica e coordenador do Projeto de Instalação do Sítio Controlado de Geofísica Rasa do IAG (Fapesp 02/07509-1).

Welitom Rodrigues Borges é geólogo pelo Instituto de Ciências Exatas e da Terra da Universidade Federal de Mato Grosso (ICET/UFMT), em 2000. Em 2002 obteve o título de Mestrado em Ciências, área de Geofísica Aplicada, pelo Instituto de Astronomia, Geofísica e Ciências Atmostéricas da Universidade de São Paulo (IAG/USP). Atualmente está fazendo o Doutorado (bolsista Fapesp 02/07510-0) junto ao Programa de Pós-Graduação em Geofísica do IAG/USP, desenvolvendo a pesquisa Caracterização geofísica de alvos rasos com aplicações no planejamento urbano, meio ambiente e arqueologia: estudo sobre o sítio controlado do IAG/USP. 